(2) Open Access Full Text Article

\title{
Comparison of classical diagnostic criteria and Chinese revised diagnostic criteria for fever of unknown origin in Chinese patients
}

This article was published in the following Dove Press journal:

Therapeutics and Clinical Risk Management

12 October 2016

Number of times this article has been viewed

\author{
Jia-Jun Li \\ Wen-Xiang Huang \\ Zheng-Yu Shi \\ Qiu Sun \\ Xiao-Juan Xin \\ Jin-Qiu Zhao \\ Zhen Yin
}

Department of Infectious Diseases,

The First Affiliated Hospital of

Chongqing Medical University,

Chongqing, People's Republic of China

Correspondence: Wen-Xiang Huang Department of Infectious Diseases, The First Affiliated Hospital of Chongqing Medical University, No I Friendship Road, Yuanjiagang, Yuzhong District, Chongqing 400016, People's Republic of China

Tel +8623890 I 2427

Fax +86 2389012430

Email huangwxian@yeah.net
Background: Fever of unknown origin (FUO) has always been a challenging problem for physicians since it was first reported half a century ago. This study aimed to investigate the clinical features of FUO and to compare the clinical significance of the classical diagnostic criteria and the Chinese revised diagnostic criteria of FUO.

Methods: We retrospectively collected a series of 140 patients admitted to our hospital between September 2011 and June 2013 because of prolonged febrile illnesses (lasting at least 2 weeks, temperature $\geq 38.5^{\circ} \mathrm{C}$ ) without diagnosis and categorized them into two groups according to the Chinese revised diagnostic criteria (group A) and classical diagnostic criteria (group B) for FUO. The A group included patients presenting with fever persisting between 2 and 3 weeks with the diagnosis remaining uncertain after three outpatient visits or at least 3 days of hospital investigation. The B group included patients presenting with fever persisting for more than 3 weeks with no established diagnosis after 1 week of hospital investigation. The general conditions, etiologies, definite diagnosis times, and diagnostic methods of the two groups were compared.

Results: There were no significant differences in the general conditions, etiologies, definite diagnosis times, and diagnostic methods between the Chinese revised diagnostic criteria and classical diagnostic criteria.

Conclusion: Both the examined FUO diagnostic criteria are suitable for clinical practice in this region.

Keywords: fever of unknown origin, diagnostic criteria, comparison, drug design

\section{Introduction}

Fever of unknown origin (FUO) has always been a challenging problem for physicians since it was first reported half a century ago. In 1961, Petersdorf and Beeson defined FUO as an illness of more than 3 weeks in duration, with fever higher than $38.3^{\circ} \mathrm{C}$ $\left(101^{\circ} \mathrm{F}\right)$ on several occasions, and no established diagnosis after 1 week of hospital investigation. ${ }^{1}$ The first two criteria of the definition are to exclude the most acute, self-limited, easily diagnosed diseases such as common viral illnesses. The third criterion allows sufficient time to complete the initial investigation into the febrile illness. During the past 50 years, advancements in medical technologies and epidemiological changes have altered the spectrum of febrile illnesses. Some researchers have suggested the criteria of FUO be updated to respond to the evolving trends in clinical practice. In 1991, Durack and Street suggested modifying the third criterion as follows: "the diagnosis remains uncertain after at least three outpatient visits or at least 3 days of hospital investigation" (regarded as the "classical diagnostic criteria" 
in this study). ${ }^{2}$ In 1998, Chinese researchers proposed revised FUO criteria to reflect the domestic situation - fever persisting for at least $2-3$ weeks, temperature above $38.5^{\circ} \mathrm{C}$, and an inability to establish a diagnosis after appropriate investigation, including history collection, physical examination, and auxiliary examination (specifically with regard to length of investigations compared to the classical diagnostic criteria and regarded as "Chinese revised diagnostic criteria"). ${ }^{3}$ This revision has not been verified in clinical research. The etiology spectrum of FUO and the number of patients may change radically with the decreasing fever duration and timing of the investigation, and these changes may confuse the diagnostic strategy and waste medical resources. Our study was intended to compare the differences of the two diagnostic criteria of FUO and to investigate the clinical features of FUO.

\section{Materials and methods}

From September 2011 to June 2013, we retrospectively collected a series of 140 patients admitted to our hospital who had a fever lasting 2 weeks or more, a temperature higher than $38.5^{\circ} \mathrm{C}$, and for whom diagnosis could not been established after an appropriate initial evaluation. The initial evaluation included comprehensive history collection and physical examination, complete blood cell count, differential urine analysis and microscopy, blood chemistry, including liver and renal function, chest radiography, and abdominal ultrasonography. The patients were categorized into two groups according to the following criteria: 1) A group (classical diagnostic criteria, which was modified by Durack and Street) with fever persisting for 2-3 weeks and uncertain diagnosis after three outpatient visits or at least 3 days of hospital investigation and 2) B group (Chinese revised diagnostic criteria), fever persisting longer than 3 weeks and no established diagnosis after 1 week of hospital investigation. The general conditions, etiologies, definite diagnosis times, and diagnostic methods of the two groups were compared and the clinical features of FUO were observed. The clinical application value of particular focused FUO laboratory work-up was examined, such as chest or abdominal CT, bone marrow smear, biopsy, serum level of inflammatory markers, including white blood cell count (WBC), erythrocyte sedimentation rate (ESR), C-reactive protein (CRP), and procalcitonin (PCT). All patients were followed up for 3 months after discharge by backing to hospital for the further consultation at least one time per week or using the telephone follow-up.

In this study, the conclusive factors in the establishment of the diagnosis were divided into six methods-clinical comprehensive diagnosis, laboratory diagnosis, imaging diagnosis, bacteriological diagnosis, pathological diagnosis, and therapeutic diagnosis. A physician was invited to review the information for each patient and demarcate into six categories with the same criteria. This study was performed with the approval of the ethical committee of the First Affiliated Hospital of Chongqing Medical University. All the 140 patients participated in this study gave their consents and approved this study.

The values of normal distributed data are presented as mean \pm SD. Data processing and statistical analysis were performed using the SPSS 18.0 program. Parametric data were analyzed using a student's $t$-test, and the non-parametric count data and the unnormal distributed data were examined by using a rank sum test. The Fisher's exact was used for categorical data with $<5$ counts. $P<0.05$ was considered significant in student's $t$-test and $P<0.01$ was considered significant in rank sum test.

\section{Results}

Three-hundred and seventy-nine patients were admitted to our hospital due to prolonged febrile illness without certain diagnosis, and 140 of the patients fulfilled the inclusion criteria. All the 140 patients were eligible for this study. Fifty patients were divided into group A and 90 into group B. As seen in Table 1, the two groups displayed no significant differences regarding age, sex ratio, and duration of hospitalization.

Eighty-nine cases were diagnosed before discharge, accounting for $64 \%$ of the total. In the confirmed cases, the most common causative disease was infection, which accounted for $32 \%$ of the total 140 cases, followed by connective tissue diseases (CTDs), miscellaneous disorders, and neoplasms, which accounted for $13 \%, 10 \%$, and $9 \%$ of the total, respectively. Ordinary bacteria and tuberculosis

Table I Comparison of the general conditions between the two groups

\begin{tabular}{lllll}
\hline Group & Group A $(\mathbf{n}=\mathbf{5 0})$ & Group B $(\mathbf{n}=\mathbf{9 0})$ & Total $(\mathbf{n}=\mathbf{I 4 0})$ & $\mathbf{P}$-value \\
\hline Male/female, $n$ & $27 / 23$ & $56 / 34$ & $83 / 57$ & 0.343 \\
Age, years & $42 \pm 18$ & $46 \pm 18$ & $44 \pm 18$ & 0.159 \\
Duration of hospitalization, days & $23 \pm 17$ & $19 \pm 19$ & $20 \pm 18$ & 0.303 \\
Duration of fever in hospital, days & $12 \pm 18$ & $11 \pm 12$ & $12 \pm 11$ & 0.654 \\
\hline
\end{tabular}

Notes: Data presented as mean \pm standard deviation unless otherwise noted. Results are in response to an independent samples $t$-test. 
were the common causative infectious disease agents and the respiratory tract was the most common infectious site. Hematological malignancy was more common than malignant solid tumor in the neoplastic diseases. Adult-onset Still's disease was the most common cause of CTDs. In addition, $36 \%$ of the cases remained undiagnosed at discharge and the ratio was higher than in any of the other diagnosis categories.
A comparison of the proportion of the etiologies between the two groups revealed no significant difference, as seen in Table 2.

The average definite diagnosis time is 11.4 days after the patient was enrolled. In addition, 38 in 89 confirmed cases were diagnosed after 7 days post-enrollment and accounted for $43 \%$ of the confirmed cases. There was no significant

Table 2 Comparison of the final diagnoses between the two groups

\begin{tabular}{|c|c|c|c|c|}
\hline Etiology & Group A & Group B & Total & $P$-value \\
\hline Infections & 17 cases $(34 \%)$ & $28(31 \%)$ & 45 (32\%) & 0.726 \\
\hline General bacterial infections & 7 & 18 & 25 & \\
\hline Respiratory tract infections & I & 8 & 9 & \\
\hline Biliary tract infections & I & 2 & 3 & \\
\hline Urinary tract infections & 0 & 2 & 2 & \\
\hline Septicemia & 1 & 4 & 5 & \\
\hline Hepatapostema & 2 & 0 & 2 & \\
\hline Intra-abdominal abscess & I & I & 2 & \\
\hline Infectious endocarditis & I & 0 & I & \\
\hline Typhoid fever & 0 & I & I & \\
\hline Tuberculosis & 6 & 7 & 13 & \\
\hline Pulmonary tuberculosis & 2 & 1 & 3 & \\
\hline Miliary tuberculosis & 3 & 2 & 5 & \\
\hline Tuberculous pleurisy & I & 0 & I & \\
\hline Spinal tuberculosis & 0 & 3 & 3 & \\
\hline Pelvic tuberculosis & 0 & I & I & \\
\hline Viral infections & 3 & 0 & 3 & \\
\hline Infectious mononucleosis & 2 & 0 & 2 & \\
\hline Parvovirus infection & I & 0 & I & \\
\hline Fungal infections & 0 & I & I & \\
\hline Parasitic infections & I & 2 & 3 & \\
\hline Kala-azar & I & I & 2 & \\
\hline Malaria & 0 & I & 1 & \\
\hline Neoplasms & $4(8 \%)$ & $8(9 \%)$ & $12(9 \%)$ & 0.857 \\
\hline Hematological malignancy & 2 & 6 & 8 & \\
\hline Non-Hodgkin's lymphoma & 2 & 3 & 5 & \\
\hline Leukemia & 0 & 3 & 3 & \\
\hline Malignant solid tumor & 2 & 2 & 4 & \\
\hline Hepatoma & 0 & I & I & \\
\hline Malignant peritoneal mesothelioma & I & 0 & I & \\
\hline Malignancy of undefined primary origin & I & I & 2 & \\
\hline Connective tissue diseases & $7(14 \%)$ & II (I2\%) & $18(13 \%)$ & 0.763 \\
\hline Adult-onset Still's disease & 7 & 4 & 11 & \\
\hline Systemic lupus erythematosus & 0 & 2 & 2 & \\
\hline Mixed connective tissue disease & 0 & I & I & \\
\hline Ankylosing spondylitis & 0 & I & I & \\
\hline Microscopic polyangiitis & 0 & I & I & \\
\hline Still's disease & 0 & I & I & \\
\hline Erythema nodosum & 0 & I & I & \\
\hline Miscellaneous - other disorders & $6(12 \%)$ & $8(9 \%)$ & $14(10 \%)$ & 0.557 \\
\hline Drug fever & 0 & I & I & \\
\hline Necrotizing lymphadenitis & I & I & 2 & \\
\hline Subacute thyroiditis & 4 & 4 & 8 & \\
\hline Hashimoto's thyroiditis & 0 & I & I & \\
\hline Autoimmune liver diseases & 0 & I & I & \\
\hline Myocarditis & I & 0 & I & \\
\hline Undiagnosed & $16(32 \%)$ & 35 (39\%) & $5 \mathrm{I}(36 \%)$ & 0.417 \\
\hline Total & $50(100 \%)$ & $90(100 \%)$ & $140(100 \%)$ & \\
\hline
\end{tabular}


Table 3 Comparison of the definite diagnosis times between the two groups

\begin{tabular}{|c|c|c|c|c|}
\hline Group & Group A $(n=34)$ & Group B $(n=55)$ & Total $(n=89)$ & $P$-value \\
\hline Definite diagnosis time, days ${ }^{a}$ & $10.77 \pm 10.75$ & $11.82 \pm 16.46$ & $11.42 \pm 14.48$ & 0.433 \\
\hline \multicolumn{5}{|l|}{ Distribution of diagnosis data } \\
\hline$\leq 3$ days & $8(24 \%)$ & $20(36 \%)$ & $28(31 \%)$ & 0.205 \\
\hline 4-7 days & $10(29 \%)$ & $13(24 \%)$ & $23(26 \%)$ & 0.545 \\
\hline$\geq 8$ days & $16(47 \%)$ & $22(40 \%)$ & $38(43 \%)$ & 0.513 \\
\hline
\end{tabular}

Notes: ${ }^{a}$ Data presented as mean \pm standard deviation. Results are in response to an independent samples $t$-test.

difference in the definite diagnosis times between the two groups as seen in Table 3. The average definite diagnosis time for infectious diseases is 9.5 days compared with 13.4 days for the non-infectious diseases.

The conclusive factors in the establishment of the diagnosis were divided into six methods: clinical comprehensive diagnosis, laboratory diagnosis, imaging diagnosis, bacteriological diagnosis, pathological diagnosis, and empiric therapy. There were no significant differences in conclusive diagnostic methods between the two groups as seen in Table 4. Overall, 95 patients underwent the $\mathrm{CT}$ scans for the chest or abdomen when focal signs were found in the patients. The sensitivity and specificity of the chest or abdominal CT scans for the FUO were $41.18 \%$ and $97.73 \%$, respectively. Bone marrow smears for the FUO diagnosis showed $13.89 \%$ sensitivity and $92.6 \%$ specificity in 63 cases. Biopsies for the FUO diagnosis showed $69.23 \%$ sensitivity and $100 \%$ specificity in 16 cases.

On the basis of the subtypes of FUO proposed by Durack and Street in 1991, four cases fell within HIV-related FUO, two cases fell within immune-deficient FUO, and no case fell within nosocomial FUO. The most common fever type in the confirmed cases was irregular fever (in which periods of fever and normal body temperature alternate and last approximately 5-7 days each) in 52 cases, followed by intermittent fever (in which at least once during a 24-hour period the fever spikes are separated by a return to normal body temperature) in 22 cases, remittent fever (in which elevated body temperature shows fluctuations each day but never returns to normal) in 14 cases, and continued fever (fever lasting for more than 24 hours without significant variation or any return to normal body temperature) in one case. Table 5 lists the various symptoms found in the diagnostic categories. Most of the hepatosplenomegaly was mild or moderate, and the only two severe cases were Kala-azar and non-Hodgkin's lymphoma. Localized lymphadenectasis was more common than generalized lymphadenectasis and the cervical lymph node was the most commonly involved, followed by the axillary, inguinal, and supraclavicular nodes.

The inflammatory markers, including WBC, ESR, CRP, and PCT, were examined when the patients were enrolled. As seen in Table 6, the level of these markers presented different degrees of increase in most cases. The level of WBC was more significantly increased in the CTDs than the infectious diseases $(P=0.011)$ and miscellaneous disorders $(P=0.026)$. The neoplasms $(P=0.002)$ and CTDs $(P=0.013)$ had a significantly higher level of ESR than the infectious diseases. The miscellaneous disorders had a significantly lower level of PCT than the infections $(P=0.007)$, neoplasms ( $P=0.002)$, and CTDs $(P=0.012)$. CRP was not significantly different among the four categories.

Of the 51 patients who remained undiagnosed when they were discharged, 16 were in group A and 35 were in group B. As seen in Table 7, there were no significant differences in the condition of the undiagnosed cases at the time of discharge between the two groups.

\section{Discussion}

Half a century has passed since Petersdorf and Beeson proposed the definition of FUO, and many former causes of FUO are now able to be confirmed in a shorter time due to remarkably improved medical laboratory and imaging techniques. In addition, the spectrum of FUO has been somewhat changed over the past 50 years. ${ }^{1}$ We reviewed a previous report about

Table 4 Comparison of the conclusive diagnostic methods between the two groups

\begin{tabular}{lllllll}
\hline Group & \multicolumn{2}{l}{ Conclusive diagnostic method } & & & \\
\cline { 2 - 7 } & Clinical & Laboratory & Imaging & Bacteriological & Pathological & Therapeutical \\
\hline Group A $(\mathrm{n}=34)$ & $10(31 \%)$ & $6(18 \%)$ & $10(30 \%)$ & $1(3 \%)$ & $6(15 \%)$ & $1(3 \%)$ \\
Group B $(\mathrm{n}=55)$ & $17(31 \%)$ & $5(9 \%)$ & $16(29 \%)$ & $4(7 \%)$ & $13(24 \%)$ & $0(0 \%)$ \\
Total $(\mathrm{n}=89)$ & $27(31 \%)$ & $11(12 \%)$ & $26(29 \%)$ & $5(6 \%)$ & $19(21 \%)$ & $1(1 \%)$ \\
$P$-value & 0.881 & 0.233 & 0.947 & 0.389 & 0.503 & 0.201 \\
\hline
\end{tabular}


Table 5 Common symptoms found in the diagnostic categories

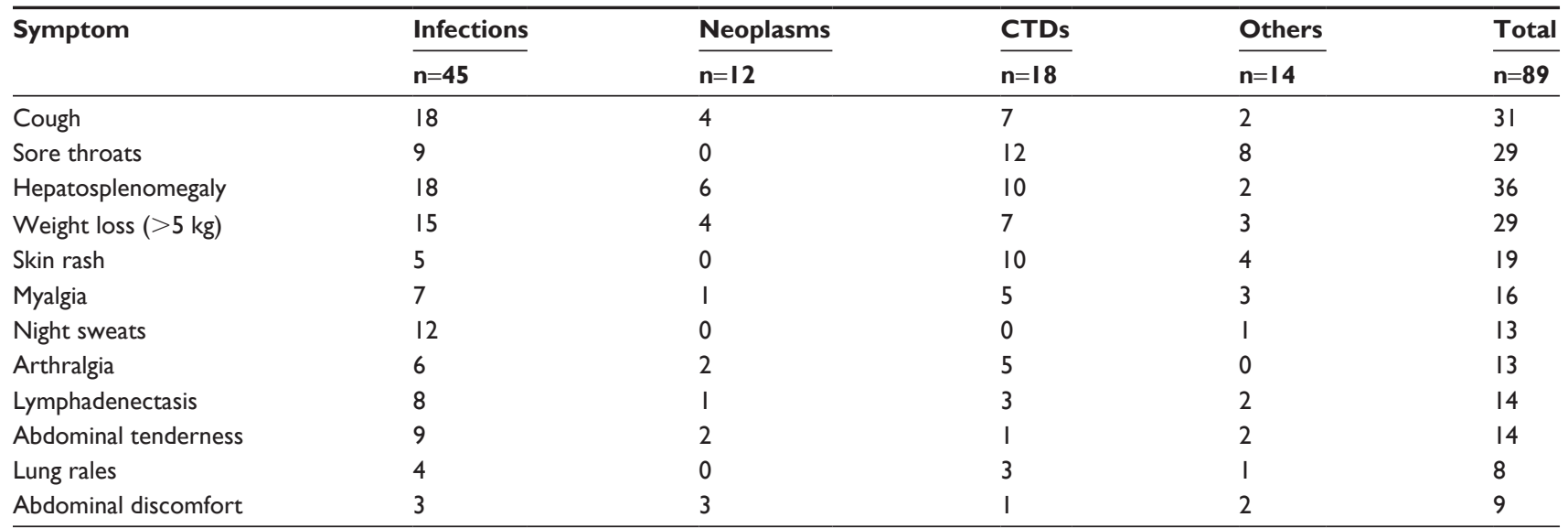

Abbreviation: CTD, connective tissue disease.

FUO in our hospital over the last 20 years that indicated the proportion of infections was declining year by year, whereas the proportion of CTDs and undiagnosed illnesses increased. ${ }^{11}$ This result is consistent with other reported data. ${ }^{6,12}$ Therefore, some modifications of the definitions of FUO have been successively proposed to reflect actual clinical practice. In our study, we compared the Chinese revised FUO criteria to the classical criteria. The results indicated that there was no significant difference in the general conditions, etiologies, definite diagnosis times, and diagnostic methods between the two criteria. Therefore, we consider that physicians can adopt the same diagnostic strategies for both FUO criteria. The Chinese revised criteria are as suitable for clinical practice in this region as the classical criteria.

As shown in the earlier results, the etiology spectrum of FUO is composed of common diseases, which is similar to most previous studies on FUO, such as infections, neoplasms, CTDs, miscellaneous disorders, and undiagnosed illnesses. ${ }^{1,4-10}$ Accordingly, it is difficult to establish the diagnosis in the initial evaluation due to two main reasons: most cases lack typical clinical characters in the initial stage or even throughout the course of diseases or some important detail was ignored by the physicians at the initial visit.
In our study, five cases of miliary tuberculosis had received chest X-ray during the 2 weeks before admission, but no abnormal results had been found, and the CT scan displayed the imaging features of miliary tuberculosis in the patients after hospitalization. One case of infective endocarditis had an absence of heart murmurs and ultrasonic imaging findings of cardiac valve excrescence, but the definite diagnosis was established by surgical biopsy. In addition, four in five cases of non-Hodgkin's lymphoma were not found with superficial lymphadenectasis, and three of these cases were confirmed by surgical biopsy of deep-located lesion. In another case, the lymphoma cells were not observed consistently until the third bone marrow puncture. In one case of Kala-azar, the diagnosis had not been established during year-long bouts of repeated fever before admission. We examined the medical history in detail and found that the patient had been to an endemic area many times during the half year preceding the onset of illness. Leishman-Donovan body was detected by bone marrow smear, and the temperature and other symptoms recovered soon after treatment with sodium stibogluconate. The number of subacute thyroiditis cases was slightly unexpectedly large. In most of these cases, thyroid tests were neglected at outpatient visits.

Table 6 Inflammatory markers among the diagnostic categories

\begin{tabular}{lllll}
\hline Diagnostic categories & WBC $\left(\times 10^{9} / \mathbf{L}\right)$ & ESR $(\mathbf{m m} / \mathbf{h})$ & CRP $(\mathbf{m g} / \mathbf{L})$ & PCT $(\mathbf{n g} / \mathbf{m L})$ \\
\hline Infections $(\mathrm{n}=45)$ & $9.11 \pm 5.76$ & $53.77 \pm 43.23$ & $95.76 \pm 76.45$ & $1.72 \pm 2.96^{\#}$ \\
Neoplasms $(\mathrm{n}=12)$ & $13.56 \pm 12.53$ & $111.9 \pm 47.91^{*}$ & $160.94 \pm 101.64$ & $0.95 \pm 1.57^{\#}$ \\
CTDs $(\mathrm{n}=18)$ & $13.55 \pm 6.95^{*}$ & $87.77 \pm 30.42^{*}$ & $110.97 \pm 60.92$ & $0.69 \pm 1.05^{\#}$ \\
Miscellaneous disorders $(\mathrm{n}=15)$ & $8.31 \pm 4.08$ & $75.5 \pm 37.4$ & $70.11 \pm 61.32$ & $0.23 \pm 0.39$ \\
Total $(\mathrm{n}=89)$ & $10.51 \pm 7.46$ & $71.77 \pm 46.11$ & $102.12 \pm 78.7$ & $1.15 \pm 2.29$ \\
\hline
\end{tabular}

Notes: *P-value represents the index in the groups compared to "infections" group. "P-value represents the index in the groups compared to miscellaneous disorders group.

Abbreviations: CTD, connective tissue disease; WBC, white blood cell count; ESR, erythrocyte sedimentation rate; CRP, C-reactive protein; PCT, procalcitonin. 
Table 7 Comparison of the discharge conditions of undiagnosed cases between two groups

\begin{tabular}{llll}
\hline Groups & $\begin{array}{l}\text { Temperature } \\
\text { recovery }\end{array}$ & $\begin{array}{l}\text { Still } \\
\text { febrile }\end{array}$ & Dead \\
\hline Groups A $(n=16)$ & $6(38 \%)$ & $9(58 \%)$ & I $(6 \%)$ \\
Groups B $(n=35)$ & $13(37 \%)$ & $22(63 \%)$ & $0(0 \%)$ \\
Total $(n=51)$ & $19(37 \%)$ & $31(61 \%)$ & I $(2 \%)$ \\
P-value & 0.98 & 0.654 & 0.135 \\
\hline
\end{tabular}

A few previous studies evaluated the clinical value of some examinations in FUO. ${ }^{7}{ }^{12-14}$ Systematic, detailed history collection and physical examination are widely considered to be irreplaceable. Sufficient time to observe the disease development and repeating some routine examination are very important to obtaining new clues that lead to establishing the diagnosis. Blood and bone marrow cultures display a low diagnostic yield in FUO. Imaging tests, including X-rays, CT scans, and ultrasonography, are very helpful for finding and localizing the lesions of abscesses and tumors. In our study, chest and abdomen CT scans, bone marrow smears, and biopsies displayed a relatively low sensitivity but a high specificity. These findings are consistent with the results of other studies. ${ }^{12,13}$ We affirm the important role of these three focused evaluations due to their specific diagnostic value for some common causes of FUO such as abscesses, hematological malignancy, and solid tumors.

The levels of inflammatory markers, including WBC, ESR, CRP, and PCT, had varying degrees of increase in different cause categories; at least one of these markers presented abnormal increases in $98.6 \%$ of all patients and all of the four markers presented abnormal increases in $72.2 \%$ of the CTDs. As mentioned, the WBC level was significantly increased in the CTDs compared with the infectious diseases and miscellaneous disorders. This is because most adult-onset Still's have WBC increase. Remarkably, increased WBC is an important presentation of adult-onset Still's disease. ${ }^{15}$ ESR displayed a higher level in the neoplasms and CTDs than the infectious diseases, and PCT displayed a lower level in the miscellaneous disorders compared with infections, neoplasms, and CTDs. The differences in the levels of inflammatory markers could provide references for initial identification of the etiological categories of FUO, but these laboratory indices are influenced by many factors, and the other study result was not consistent with our study. ${ }^{15}$ Thus, the predictive value of these inflammatory marker levels in the evaluation of FUO is still not definite..$^{12-14,16}$

Cases representing three subtypes of FUO, including nosocomial FUO, immune-deficient FUO, and HIV-related FUO, as proposed by Durack and Street in 1991, were lacking in numbers in our study, and therefore, we did not further analyze their clinical features. The vast majority of the patients in our study were from the Chongqing area. Therefore, the results of this study need to be replicated by further studies to confirm whether the results apply to other areas.

\section{Conclusion}

In summary, there were no significant differences in the general conditions, etiologies, definite diagnosis times, and diagnostic methods between the Chinese revised diagnostic criteria and classical diagnostic criteria. In conclusion, both the examined FUO diagnostic criteria are suitable for clinical practice in this region.

\section{Disclosure}

The authors report no conflicts of interest in this work.

\section{References}

1. Petersdorf R, Beeson P. Fever of unexplained origin: report on 100 cases. Medicine. 1961;40:1-30

2. Druack DT, Street AC. Fever of unknown origin-reexamined and redefined. Curt Clin Top Brect Dis. 1991;11:35-51.

3. Alavi SM, Nadimi M, Zamani GA. Changing pattern of infectious etiology of fever of unknown origin (FUO) in adult patients in Ahvaz, Iran. Caspian J Intern Med. 2013;4:722-726.

4. Zhou W, Tan X, Li Y, Tan W. Human herpes viruses are associated with classic fever of unknown origin (FUO) in Beijing patients. PLoS One. 2014;9:e101619.

5. Alpa M, Roccatello D. Canakinumab as rescue therapy in familial Mediterranean fever refractory to conventional treatment. Drug Des Devel Ther. 2015;9:1983-1987.

6. Ikuni Y, Okada J, Knodo H, Kashiwazaki S. Current fever of unknown origin, 1982-1992. Int Med. 1994;33:67-73.

7. Quesada AE, Tholpady A, Wanger A, Nguyen AN, Chen L. Utility of bone marrow examination for workup of fever of unknown origin in patients with HSV/AIDS. J Clin Pathol. 2015;68:241-245.

8. Arce-Salinas C, Morales-Velázquez J, Villaseñor-Ovies $\mathrm{P}$, et al. Classical fever of unknown origin (FUO): current causes in Mexico. Rev Invest Clin. 2005;57:762-769.

9. Hersch EC, Oh RC. Prolonged febrile illness and fever of unknown origin in adults. Am Fam Physician. 2014;90:91-96.

10. Tokmak H, Ergonul O, Demirkol O, Cetiner M, Ferhanoglu B. Diagnostic contribution of (18)F-FDG-PET/CT in fever of unknown origin. Int J Infect Dis. 2014;19:53-58.

11. Zhuo C, Wang Q, Huang W. A clinical review of 184 cases with fever of unknown origin. Chin J Practical Intern Med. 2006;26:312-315.

12. Mourad O, Palda V, Detsky A. A comprehensive evidence-based approach to fever of unknown origin. Arch Int Med. 2003;163:545-551.

13. Cunha BA. Fever of unknown origin: focused diagnostic approach based on clinical clues from the history, physical examination, and laboratory tests. Infect Dis Clin North Am. 2007;21:1137-1187.

14. Efstathiou SP, Pefanis AV, Tsiakou AG, et al. Fever of unknown origin: discrimination between infectious and non-infectious causes. Eur J Intern Med. 2010;21:137-143.

15. Vanderschueren S, Eyckmans T, De Munter P, Knockaert D. Mortality in patients presenting with fever of unknown origin. Acta Clin Belg. 2014; 69:12-16.

16. Zhang L, Zheng JW, Yuan WE. Treatment of alarming head and neck infantile hemangiomas with interferon-alpha-2a: a clinical study in eleven consecutive patients. Drug Des Devel Ther. 2015;9:713-727. 
Therapeutics and Clinical Risk Management

Dovepress

\section{Publish your work in this journal}

Therapeutics and Clinical Risk Management is an international, peerreviewed journal of clinical therapeutics and risk management, focusing on concise rapid reporting of clinical studies in all therapeutic areas, outcomes, safety, and programs for the effective, safe, and sustained use of medicines. This journal is indexed on PubMed Central, CAS,

EMBase, Scopus and the Elsevier Bibliographic databases. The manuscript management system is completely online and includes a very quick and fair peer-review system, which is all easy to use. Visit http://www.dovepress.com/testimonials.php to read real quotes from published authors.

Submit your manuscript here: http://www.dovepress.com/therapeutics-and-clinical-risk-management-journal 